\title{
Fight for Beauty! Un Pentalogo
}

\author{
di Tomaso Kemeny \\ kemeny@libero.it
}

Il Decalogo designa i Dieci Comandamenti di Dio, le tavole della legge morale. Il mio Pentalogo propone una normativa estetica in cinque punti.

Se Albert Camus afferma che la bellezza, senza dubbio, non fa rivoluzione, il pentalogo propone forme di Bellezza in grado di cambiare la vita dei singoli e del mondo. Oscar Wilde afferma che la Bellezza è l'unica che resiste al tempo.

Dal mio punto di vista ha più ragione Esiodo quando afferma che la bellezza è quella che ci introduce ad un nuovo modo di vedere la realtà.

La Bellezza non ha canoni, né modelli come affermò Baudelaire: «Ci sono tanti tipi di Bellezza quanti sono i modi di cercare la felicità».

Ha ragione Socrate quando affermò che «La bellezza è difficile?».

Non oggi, quando la bellezza occhieggia dalle vetrine dei miti del consumismo; e aveva ragione Shakespeare quando affermò che «la Bellezza attira i ladri più dell'oro».

Sono grato a Friedrich von Schiller quando prospetta l'unità del mondo sotto le bandiere della Bellezza.

Alla Morte di Andrè Breton (1968) scrissi un poemetto che si conclude con il distico «Quando coloro che fanno professione rivoluzionaria / sapranno salutare la bellezza».

È ovvio che il mio "Fight for Beauty" è un invito a combattere, poiché oggi viene messo in gioco la speranza, l'utopia, i reami di una realtà nuova. Perciò lo slogan "Fight for Beauty" è una forma di fondamentalismo estetico.

L'ideale della bellezza ha radici in Europa: «Bellezza dell'Europa, ti voglio fino alla fine», come scrisse il poeta Giuseppe Conte. 
Negli anni 80, il movimento Mitomodernista, ispirato dal filosofo Stefano Zecchi ha vergato 19 tesi sulla vita della bellezza. Mentre oggi è stata sdoganata l'idea di Bellezza, il mio pentalogo servirà a determinare l'orizzonte della ricerca secondo valori mitomodernisti. Fu nel 1994 che Stefano Zecchi e Giuseppe Conte fecero salpare il vascello del Mitomodernismo, vascello su cui viaggio anch'io sugli oceani della bellezza e del destino mitico.

La mia rivolta si basa su tre "Er": "Eresia", ovvero il sacro oltre le religioni rivelate; "Eroismo" oltre le ideologie storiche; "Erotismo", il sesso inscindibile da valori estetici.

Il vascello del Mitomodernismo, inoltre, sfida tutte le ingiustizie sociali, la svalutazione del contributo femminile, il razzismo. Si tratta del tentativo di energizzare il verbo poetico, trasformandolo in atti.

La politica abbia il primato sull'economia, la poesia sulla politica.

Contro la brutalità contemporanea vincano le ragioni della poesia e dell'estetica, si deve seminare il meraviglioso nell'oscurità della realtà.

La battaglia abbia come "parole d'ordine" Bellezza e Mito.

Proporre pensieri oltre il Marxismo sociologico. Rivalutare i valori che portano al sublime. La proposta della bellezza come valore universale deriva dalla riscoperta dello stupore cosmico di fronte all'universo.

Il mio Pentalogo è la negazione della bellezza come cosa perduta nella casa del desiderio, come nostalgia della perfezione arcaica; INVECE si tratta di un ideale che solo è in grado di produrre la tensione simbolica dell'origine dell'autenticità, quella che apre al dubbio e all'ideale della ricerca permanente.

La Bellezza al suo apparire è sempre verginale, immune al passare del tempo. Allo stesso tempo si concede alla metamorfosi, non è oggetto della nostalgia dell'armonia della perfezione classica. Essa apre all'azione in grado di realizzare una struttura ideale della realtà.

In favore della Bellezza è inevitabile l'autonomia dell'arte e della poesia perché possano parlare al mondo in un linguaggio che sia contemporaneo. 
La Bellezza è anche un valore Etico. Il Brutto fiorisce dal male e da tutto ciò che è negativo.

L'energia del linguaggio simbolico agisce sull'immaginario che favorisce riduzione dell'autentico nel tempo storico-sociale.

La Bellezza in poesia è in grado di fondere la categoria del sublime con l'animo dell'individuo come tale.

La bellezza ha l'effetto di farci sentire più grandi di quel che siamo, tanto da farci sentire sollevati da terra e l'euforia ed il senso di liberazione sono tali, che tutto il resto non ha più importanza. Ci si trova sradicati in un mondo di puro spirito.

Quando il poeta viene colto dall'ispirazione grazie alla quale le idee e le parole gli vengono non si sa da dove, è allora che scopre ciò che non sapeva di sapere, vive una condizione indistinguibile da quella del mistico in estasi.

Esempio: «Naufragar mi è dolce in questo mare» (Giacomo Leopardi).

\section{Nota biografica}

Tomaso Kemeny (Budapest 1938), vive a Milano dal 1948. In qualità di anglista, professore ordinario presso l'Università di Pavia, ha scritto libri, saggi e articoli sull'opera di C. Marlowe, S. T. Coleridge, P. B. Shelley, Lord Byron, Lewis Carroll, Dylan Thomas, James Joyce e Ezra Pound. Ha pubblicato undici libri di poesia tra cui Il guanto del sicario (1976), Il libro dell'Angelo (1991), La Transilvania liberata (2005), Poemetto gastronomico e altri nutrimenti (2012), 107 incontri con la prosa e la poesia (2014). Boomerang (2018). Ha scritto libri di poetica come L'arte di non morire (2000) e Dialogo sulla poesia (con Fulvio Papi, 1997); il romanzo Don Giovanni innamorato (1993); il testo drammatico La conquista della scena e del mondo (1996). Con Cesare Viviani ha organizzato i seminari sulla poesia degli anni '70 presso il Club Turati di Milano (1978-79). Tra le sue curatele La dicibilità del sublime (con E. C. Ramusino, 1989), Le avventure della bellezza, 1988- 
2008 (2008). È tra i fondatori del movimento internazionale mitomodernista (1994) e del movimento "Poetry and Discovery" (2016) nonché della Casa della poesia di Milano (2006). Tra i pubblici riconoscimenti: il "Premio speciale per la traduzione Giuseppe Acerbi"(2006), il "Montale fuori mura, alla carriera" (2015) e il "Premio Genesi per la Carriera" (2019). 